\title{
Safety and effectiveness of oral propranolol for infantile hemangiomas started before 5 weeks and after 5 months of age: an Italian multicenter experience
}

\author{
Maya El Hachem ${ }^{1}$, Francesco Gesualdo ${ }^{2^{*}}$ D , Andrea Diociaiuti ${ }^{1}$, Irene Berti ${ }^{3}$, Nadia Vercellino ${ }^{4}$, Valeria Boccaletti ${ }^{5}$, \\ Iria Neri ${ }^{6}$, Giulio Porcedda ${ }^{7}$, Antonella Greco ${ }^{7}$, Claudia Carnevale ${ }^{1}$, Teresa Oranges ${ }^{1}$, Mario Cutrone ${ }^{8}$ \\ and Pietro Dalmonte ${ }^{4}$
}

\begin{abstract}
Background: Despite not being licensed for the treatment of infantile hemangiomas $(\mathrm{IH})$ in infants younger than 5 weeks or older than 5 months, propranolol is often used in these age groups to prevent or to treat potentially severe complications. The objective of the present study was to review the experience of 8 Italian pediatric and dermatologic centers regarding propranolol treatment for $\mathrm{IH}$ started before 5 weeks or after 5 months of age.

Methods: We retrospectively reviewed the records of patients followed up for $\mathrm{IH}$, on propranolol treatment started before 5 weeks or after 5 months of age, and collected information on sociodemographic data, treatment indications, $\mathrm{IH}$ involution, $\mathrm{IH}$ relapse, and treatment side effects.

Results: A total of 343 patients were enrolled; 15 were started on propranolol before 5 weeks (group 1), 328 were started after 5 months of age (group 2). The most frequent indications were permanent aesthetical disfigurement (91. $8 \%$ ) and function threatening complications (42.6\%).

In most cases, the treatment was effective. The involution was partial in $67.7 \%$ of patients. In $11.8 \%$ of cases a relapse was observed. No relapse was observed in group 1. Treatment complications were reported in $15.8 \%$ of children, most frequently sleep disorders (6.6\%), followed by irritability (5.1\%) and diarrhea (2.2\%). Only a case of mild constipation was observed in group 1.

Conclusion: The safety and effectiveness profile of propranolol in infants younger than 5 weeks or older than 5 months may be acceptable. Taking in account propranolol's potential in preventing severe complications, further studies should assess the acceptability of propranolol treatment, especially in the $<5$-week age group .
\end{abstract}

\section{Background}

Infantile hemangiomas (IHs) are the most common benign neoplasms in infancy [1-5]. The natural history of IHs is characterized by an onset within the first weeks of life, followed by a proliferative phase with rapid growth, generally lasting until 5 months of age; in some cases, the proliferative phase may last up to 10-11 months [6].

\footnotetext{
* Correspondence: f.gesualdo@gmail.com

${ }^{2}$ Multifactorial and Complex Disease Research Area, Bambino Gesù Children's Hospital IRCCS, Viale Baldelli 41, 00146 Rome, Italy

Full list of author information is available at the end of the article
}

About $90 \%$ of IHs spontaneously regress, partially or completely, within the 7th year of life (usually between the 3rd and the 6th year) $[2,7,8]$. In $10-15 \%$ of cases, a treatment is required, with the following indications: life threatening complications, function threatening complications, ulceration not responding to local treatments, pain and potential permanent disfigurement [6, 9-15].

Oral propranolol has been repeatedly demonstrated as the first-choice drug for $\mathrm{IH}$ and, at present, is available in several countries with a specific indication for pediatric patients $[6,16,17]$. 
The treatment is highly effective (up to $96-98 \%$ after 6 months) and has an optimal safety profile, with a low incidence of adverse events, such as sleep disorders, diarrhea, cold extremities, irritability, hypotension, bradycardia, bronchospasm and hypoglycemia. Symptomatic hypotension and bradycardia are very rare [17-19]. All side effects are usually transient and only in few cases a definitive treatment discontinuation is required [20].

According to international consensus recommendations and to the drug's summary of product characteristics (SmPC), oral propranolol should be administered in infants between 5 weeks and 5 months of age, for a duration shorter than 6 months [6]. Nevertheless, peculiar clinical pictures may exist, in which an off-label treatment could be required or suggested.

The aim of our study was to describe the experience of 8 Italian pediatric and dermatologic centers in the off-label use of oral propranolol for the treatment of $\mathrm{IH}$, starting earlier than 5 weeks or later than 5 months of age, in terms of treatment indications, effectiveness and side effects.

\section{Methods}

\section{Study design and population}

This is a multicenter, retrospective cohort study that was conducted in the Pediatric Units and Pediatric Dermatology Units of 8 major Italian metropolitan hospitals.

We retrospectively reviewed the records of patients followed up for $\mathrm{IH}$ in the participating centers between June, 2008 and June, 2015, who had been treated with oral propranolol started before 5 weeks or after 5 months of age, and who had discontinued the treatment for at least one month. The following data were collected: sociodemographic data; risk factors for IH; IH therapy (indication, dose, therapy duration); treatment effectiveness (involution at treatment end, relapse); treatment complications (including irritability, nightmares, cold extremities, diarrhea, hypoglycemia, hypotension).

Data were collected in a dedicated database.

The study was approved by the Ethical Committee of the Bambino Gesù Children's Hospital.

\section{Statistical analysis}

Data were described as means and SD or medians and range or proportions and $\mathrm{CI}$, as appropriate. Age is expressed in months, and was corrected to 40 weeks of gestational age for preterm infants.

Stata 13 was used for the statistical analysis.

\section{Results}

\section{Study population}

We enrolled a total of 343 patients. Characteristics of the study population are presented in Table 1.

A total of 242 (70.8\%) were female.
Fifteen patients $(4.3 \%)$ were started on oral propranolol before 5 weeks of age; 328 patients (96\%) had started the treatment after 5 months of age.

Median age at treatment start was 0.8 months in the subgroup of patients starting the therapy before 5 weeks of age (range 0-1.1 months) and 9 months in the subgroup of patients starting the therapy after 5 months (range 5-90.7 months).

Median treatment duration was 9.5 months (range 6.3-14.5 months) among patients starting the therapy before 5 weeks of age and 6.8 months (range 0.5-20 months) in those starting the therapy after 5 months. A patient was treated with propranolol for 2 weeks only, waiting to be treated surgically. Despite the short interval between the treatment start and the surgical intervention, the clinicians decided to treat the patient anyway, in order to reduce the IH's vascularization and the consequent risk for an intraoperative hemorrhage.

A total of 57 children (16.6\%) were born preterm. The proportion of preterms among patients starting the treatment before 5 weeks of age was high (53\%).

At least one risk factor among those recognized in the medical literature $[6,21]$ and listed in Table 1 was identified in 82 patients (24\%), most commonly twin pregnancy $(5.5 \%)$, amniocentesis $(4.7 \%)$ and preeclampsia or placenta previa (4\%). Risk factors were reported in a high proportion $(60 \%)$ of patients starting the treatment before 5 weeks and in $22.3 \%$ of patients starting the treatment after 5 months. Twin pregnancy was the most common risk factor in both groups.

\section{Treatment indications and dosages}

Treatment indications are reported in Table 2 .

In both groups, the most frequent indications to treatment with propranolol was potential permanent aesthetical disfigurement, followed by function threatening complications. Other indications accounted for less than $5 \%$ in the subgroup started on propranolol after 5 months of age. On the other hand, among infants starting the treatment before 5 weeks, life threatening, ulceration and pain were reported in a total of 5 patients ( $1 / 3$ of the subgroup). Of the subgroup of 6 patients with a life threatening indication, the following localizations were reported: laryngeal (3 patients), liver (3 patients), and hemithorax (1 patient). All patients started on propranolol before 5 weeks of age for life threatening complications had a liver hemangiomatosis.

Median therapeutic dosage was $2 \mathrm{mg} / \mathrm{Kg}$ daily (range $1-3)$. A proportion of $54 \%$ patients had a dosage between 1.8 and $2.2 \mathrm{mg} / \mathrm{Kg}$. As per protocol, children $<5$ weeks were started on a lower dosage and progressively adjusted. 
Table 1 Sociodemographic characteristics and IH risk factors

\begin{tabular}{|c|c|c|c|}
\hline & Start $<5$ weeks $(N=15)$ & Start $>5$ months $(N=328)$ & Total $(N=343)$ \\
\hline Female sex (N, \%) & $10(66.7)$ & $232(71)$ & $242(70.8)$ \\
\hline Age at treatment start (median, range) & $0.8(0-1.1)$ & $9(5-90.7)$ & $8.7(0-90.7)$ \\
\hline Age at treatment end (median, range) & $10(7.3-15.2)$ & $16.1(8.6-107.1)$ & $16(7.3-107.1)$ \\
\hline Treatment duration (median, range) & $9.5(6.3-14.5)$ & $6.8(0.5-20)$ & $6.8(0.5-20)$ \\
\hline Preterm (N, \%) & $8(53.3)$ & $49(14.9)$ & $57(16.6)$ \\
\hline Risk factors (N, \%) & $9(60)$ & $73(22.3)$ & $82(24)$ \\
\hline twins & $3(20)$ & $16(4.8)$ & $19(5.5)$ \\
\hline IVF & $1(6.7)$ & $2(0.6)$ & $3(0.9)$ \\
\hline family history of IE & $0(0)$ & $9(2.7)$ & $9(2.6)$ \\
\hline amniocentesis & $1(6.7)$ & $15(4.6)$ & $16(4.7)$ \\
\hline villocentesis & $2(13.3)$ & $10(3)$ & $12(3.5)$ \\
\hline pre-eclampsia or placenta praevia & $2(13.3)$ & $12(3.7)$ & $14(4)$ \\
\hline
\end{tabular}

\section{Effectiveness and safety}

In most cases, the treatment was effective, although the involution at the end of the treatment was partial in $2 / 3$ of patients, in both groups (Table 3).

Among the 15 patients started on propranolol before 5 weeks, an involution was seen in all patients (complete: 2 patients; almost complete: 3 patients; partial: 10 patients).

Among patients started on propranolol after 5 months, a complete or almost complete involution at treatment end was observed in $23.9 \%$ and $5.3 \%$, respectively. Only in $3 \%$ of cases no involution was observed.

Relapse was infrequent among patients started on treatment before 5 weeks (recorded in 1 patient only). Twelve patients in the older group had a relapse.

Treatment complications were reported in $15.8 \%$ of children, mostly children started on propranolol after 5 months of age.

In this group, the most frequent complications were sleep disorders (6.8\%), followed by irritability (5.4\%) and diarrhea (3.1\%). Cold extremities, hypoglycemia, hypotension and wheezing were observed in a very low proportion of cases. All the described cases of hypoglicemia and hypotension were asymptomatic. A severe complication was reported in a child started on propranolol at 12 months of age for a wide $\mathrm{IH}$ of the back, with a high risk of ulceration. The patient had a cardiac arrest after 40 days of treatment, during a respiratory tract infection.
Among patients starting the treatment before 5 weeks of age, only a case of mild constipation was observed.

\section{Discussion}

With the present descriptive study, we show that the off-label use of oral propranolol for IHs in infants, with treatment start before 5 weeks or after 5 months of age, may have an acceptable profile of effectiveness and safety.

Based on the only randomized controlled trial (RCT) available in the literature [19], the indications for oral propranolol in infants suggest a treatment start between 5 weeks and 5 months.

Off-label use of propranolol after 5 months of age is frequent and already described in the literature [22].

As a matter of fact, despite children with an IH requiring treatment should be started on oral propranolol within the first 2-3 months of age, referral to dermatology centers may be late (after 5 months), due to a scarce knowledge of the therapeutic option of propranolol, and of its safety and efficacy, among general pediatricians. Moreover, despite the regression of $\mathrm{IH}$ is usually spontaneous after 1 year of age, sometimes complications may be severe and waiting for a spontaneous involution may cause irreversible functional and aesthetical damage. In infants referred to pediatric dermatology centers after 5 months of age, propranolol, through its proapoptotic properties, may promote and accelerate the

Table 2 Treatment indications

\begin{tabular}{|c|c|c|c|}
\hline & Start <5 weeks $(N=15)$ & Start >5 months $(N=328)$ & Total $(N=343)$ \\
\hline Life threatening complications & $2(13)$ & $4(1.2)$ & $6(1.8)$ \\
\hline Function threatening complications & $5(66.7)$ & $136(41.4)$ & $146(42.6)$ \\
\hline Permanent esthetical disfigurement & $13(86.7)$ & $302(92.1)$ & $315(91.8)$ \\
\hline Ulceration & $3(20)$ & $8(2.4)$ & $11(3,2)$ \\
\hline Pain & $2(13.3)$ & $4(1.2)$ & $6(1.8)$ \\
\hline
\end{tabular}


Table 3 Effectiveness and safety

\begin{tabular}{llll}
\hline & Start $<5$ weeks $(N=15)$ & Start $>5$ months $(N=328)$ & Total $(N=343)$ \\
\hline $\begin{array}{lll}\text { Involution } \\
\text { complete }\end{array}$ & $2(13.3)$ & & $77(23.9)$ \\
almost complete & $3(20)$ & $17(5.3)$ & $79(23.4)$ \\
partial & $10(66.7)$ & $218(67.7)$ & $228(5.9)$ \\
absent & 0 & $10(3.1)$ & $10(3)$ \\
Relapse & $1(6.7)$ & $39(12)$ & $40(11.8)$ \\
Treatment complications & $1(6.7)$ & $52(15.9)$ & $53(15.5)$ \\
irritability & 0 & $17(5.4)$ & $17(5.12)$ \\
sleep disorders & 0 & $22(6.8)$ & $22(6.6)$ \\
cold extremities & 0 & $2(0.6)$ & $2(0.6)$ \\
diarrhoea & 0 & $10(3.1)$ & $10(2.9)$ \\
hypoglicemia & 0 & $1(0.3)$ & $1(0.3)$ \\
hypotension & 0 & $4(1.3)$ & $4(1.2)$ \\
\hline
\end{tabular}

involution of the lesion, thus reducing the risk of permanent damages.

On the other hand, a treatment start before 5 weeks of age is less frequently reported. Although very rarely, IHs may actually pose a risk of severe and possibly irreversible complications at birth or within the first 5 weeks of life, thus justifying an early treatment start, with an indication of life or function threatening complications, eg. laryngeal hemangiomas impairing the breathing dynamic, periorbital hemangiomas entailing a risk of amblyopia and liver hemangiomas impairing the hemodynamics, with a high risk of cardiac failure. When starting the treatment before 5 weeks, special precautions should be carefully taken, accordingly to consensus guidelines $[6,23]$ : hospitalization at treatment start and during dose adjustment, low initial dose, continuous monitoring of blood pressure and heart rate for the first week, follow up visits every 2-4 weeks.

The experience of the major Italian pediatric and dermatologic centers reported in the present article confirms an acceptable profile of the drug in terms of effectiveness, safety and prevention of IH-related complications in children younger than 5 weeks and older than 5 months.

Effectiveness was very high. Any improvement (including partial, almost complete and complete involution) was reported in $97 \%$ of the study population. The authors of a systematic review published in 2013 and including 41 articles on propranolol treatment for IH reported a mean response rate of $98 \%$ (ranging from $82 \%$ to $100 \%)$, which is consistent with our figures. Nevertheless, according to this review, rebound growth and adverse events were more frequent compared to our population (rebound growth $=17 \%$ compared to $11.8 \%$ in our population; adverse events $=30 \%$ compared to $15.8 \%$ in our population) [24].
In a recent article on propranolol safety, the reported complication rate was lower $(8.8 \%)$ compared to our figures [25]. The reason of this lower proportion of treatment complications is probably due to the fact that the authors assessed the causality using a standardized protocol [26].

In our population, 15 children were started on propranolol before 5 weeks of age, mainly due to lifethreatening complications, ulceration and pain. In this group, risk factors were highly represented, especially preterm birth and twin pregnancy. Efficacy was high, as involution of the lesion was observed in all cases started on treatment before 5 weeks, and only one case of relapse was described, in a child treated for a parotideal IH. Parotideal IHs are known to exhibit an extended growth phase during the second and third year of life. This could explain the frequent relapse at treatment discontinuation $[19,27]$. As a matter of fact, the low proportion of relapse in this subgroup may be related to the fact that the treatment duration was always longer than 6 months (median 9.5 months). The SmPC suggests a treatment duration shorter than 6 months. However, our data suggest that the safety and effectiveness of a longer treatment duration in terms of reduction of relapse may be convenient and should be further investigated. Moreover, no side effect of the treatment was observed in this subgroup, apart from a mild case of constipation. We believe that the extreme accuracy of the management of these very young infants, with slow dosage increase and continuous monitoring of vital signs, has strongly contributed to the prevention of side effects.

Among the subgroup of patients started on propranolol after 5 months of age, treatment complications were reported in $16 \%$ of cases, mainly including sleep disorders and irritability. One severe adverse event was 
reported: a cardiac arrest during a respiratory tract infection in a child that had been treated for 40 days. The child suffered from breath-holding spells and was dehydrated due to the infection. Cardiac adverse events potentially related to propranolol therapy have been previously reported in the literature [25], and one case of cardiac arrest in an infant on propranolol with bronchiolitis has been described [28]. Based on these observations, parents should be carefully trained to recognize symptoms of a respiratory infection and advised to immediately discontinue treatment if a respiratory infection occurs, as previously reported [23].

The present study has several limitations, mainly with regards to its design. First of all, we did not compare our results with those obtained from a retrospective cohort of controls started on propranolol between 5 weeks and 5 months of age. Nevertheless, our results were in line with the figures reported in the literature. Moreover, causality of side effects has not been investigated with a standardized protocol, and this may have impaired our estimates, probably through an overestimation of the frequency of side effects. Finally, the subgroup of infants started on propranolol before 5 weeks of age was relatively small, and this may have affected the precision and generalizability of our estimates.

\section{Conclusion}

In conclusion, we report an acceptable profile of effectiveness and safety for the off-label use of propranolol in infants, with figures that are comparable to those reported in the literature, with regard to involution, relapse and complications. In particular, relapse and complication rates in children started on propranolol at an age younger than 5 weeks were even lower than those reported for other age groups. Despite this subgroup of patient was relatively small, this finding suggests that a closely monitored therapy start may contribute to a higher safety profile in these very young infants. On the other hand, the late start of propranolol after 5 months of age is often due to the late referral of patients by family pediatricians, which are still not aware of the availability of propranolol therapy for IH. With the aim of resolving this cultural gap, in Italy, the Italian Society for the Study of Vascular Anomalies (SISAV) and the Italian Society of Pediatric Dermatology (SIDerP) promoted educational courses dedicated to pediatricians and dermatologists for the management of IHs.

Finally, based on our observations, additional RCTs are needed to validate the use of propranolol, especially in the $<5$-week age group, in order to reduce IH-related complications, which may be severe and irreversible.
Funding

No funding.

\section{Availability of data and materials}

The datasets generated during and/or analysed during the current study are not publicly available due to hospital research policy. Aggregate analyses different from those presented in the article are however available on reasonable request to the corresponding author.

\section{Authors' contributions}

MEA, FG and AD wrote the article; IB and NV conceived the study; AG, CC and TO contributed to patient enrolment and data management; VB, IN, GP and $M C$ critically revised the article; PD revised the article and gave the final consent to publication. All authors read and approved the final manuscript.

\section{Competing interests}

The authors declare that they have no competing interests.

\section{Consent to publication}

Not applicable.

\section{Ethics approval and consent to participate}

The publication of this study was approved by the Bambino Gesù Children's Hospital Ethical Committee (protocol n.1278_OPBG_2016). Data were acquired retrospectively, therefore no consent was asked to parents of included patients.

\section{Publisher's Note}

Springer Nature remains neutral with regard to jurisdictional claims in published maps and institutional affiliations.

\section{Author details \\ ${ }^{1}$ Pediatric Dermatology Unit, Bambino Gesù Children's Hospital, IRCCS, Rome, Italy. ${ }^{2}$ Multifactorial and Complex Disease Research Area, Bambino Gesù Children's Hospital IRCCS, Viale Baldelli 41, 00146 Rome, Italy. ${ }^{3}$ Institute for Maternal and Child Health - IRCCS Burlo Garofolo, Trieste, Italy. ${ }^{4}$ Angioma Center, Cardiovascular Department, Gaslini Children's Research Institute, Genoa, Italy. ${ }^{5}$ Dermatology Clinic, Parma University Hospital, Parma, Italy. ${ }^{6}$ Division of Dermatology, Department of of Experimental, Diagnostic and Specialty Medicine, University of Bologna, Bologna, Italy. ${ }^{7}$ Pediatric Cardiology Unit, Anna Meyer Children's Hospital, Florence, Italy. ${ }^{8}$ Pediatric Emergency Department, Ospedale dell'Angelo Ulss12, Venice, Italy.}

Received: 17 January 2017 Accepted: 8 April 2017

Published online: 19 April 2017

\section{References}

1. Hoornweg MJ, Smeulders MJ, van der Horst CM. Prevalence and characteristics of haemangiomas in young children. Ned Tijdschr Geneeskd. 2005;149:44.

2. Dickison $P$, Christou E, Wargon O. A prospective study of infantile hemangiomas with a focus on incidence and risk factors. Pediatr Dermatol. 2011;28:6.

3. Leaute-Labreze C, Prey S, Ezzedine K, Leaute-Labreze C, Prey S, Ezzedine K. Infantile haemangioma: Part I. pathophysiology, epidemiology, clinical features, life cycle and associated structural abnormalities. J Eur Acad Dermatol Venereol. 2011;25:11.

4. Hoornweg MJ, Smeulders MJ, Ubbink DT, van der Horst CM. The prevalence and risk factors of infantile haemangiomas: A case-control study in the dutch population. Paediatr Perinat Epidemiol. 2012;26:2.

5. Munden A, Butschek R, Tom WL, Marshall JS, Poeltler DM, Krohne SE, et al. Prospective study of infantile haemangiomas: Incidence, clinical characteristics and association with placental anomalies. Br J Dermatol. 2014;170:4.

6. Hoeger PH, Harper Jl, Baselga E, Bonnet D, Boon LM, Ciofi Degli Atti M, et al. Treatment of infantile haemangiomas: recommendations of a european expert group. Eur J Pediatr. 2015;174:7.

7. Luu M, Frieden IJ. Haemangioma: Clinical course, complications and management. Br J Dermatol. 2013;169:1.

8. Puttgen KB. Diagnosis and management of infantile hemangiomas. Pediatr Clin North Am. 2014;61:2.

9. Chamlin SL, Haggstrom AN, Drolet BA, Baselga E, Frieden IJ, Garzon MC, et al. Multicenter prospective study of ulcerated hemangiomas. J Pediatr. 2007;151:6. 
10. Hermans DJ, Boezeman JB, Van de Kerkhof PC, Rieu PN, Van der Vleuten CJ. Differences between ulcerated and non-ulcerated hemangiomas, a retrospective study of 465 cases. Eur J Dermatol. 2009;19:2.

11. Bauland CG, Luning TH, Smit JM, Zeebregts CJ, Spauwen PH. Untreated hemangiomas: Growth pattern and residual lesions. Plast Reconstr Surg. 2011;127:4.

12. Horii KA, Drolet BA, Frieden IJ, Baselga E, Chamlin SL, Haggstrom AN, et al. Prospective study of the frequency of hepatic hemangiomas in infants with multiple cutaneous infantile hemangiomas. Pediatr Dermatol. 2011;28:3.

13. Bosemani $T$, Puttgen $K B$, Huisman $T A$, Tekes A. Multifocal infantile hepatic hemangiomas-imaging strategy and response to treatment after propranolol and steroids including review of the literature. Eur J Pediatr. 2012;171:7.

14. Chen TS, Eichenfield LF, Friedlander SF. Infantile hemangiomas: An update on pathogenesis and therapy. Pediatrics. 2013:131:1.

15. Vredenborg AD, Janmohamed SR, de Laat PC, Madern GC, Oranje AP. Multiple cutaneous infantile haemangiomas and the risk of internal haemangioma. Br J Dermatol. 2013;169:1.

16. Leaute-Labreze C, Dumas de la Roque E, Hubiche T, Boralevi F, Thambo JB, Taieb A. Propranolol for severe hemangiomas of infancy. N Engl J Med. 2008;358:24.

17. Drolet BA, Frommelt PC, Chamlin SL, Haggstrom A, Bauman NM, Chiu YE, et al. Initiation and use of propranolol for infantile hemangioma: Report of a consensus conference. Pediatrics. 2013;131:1.

18. Haggstrom AN, Drolet BA, Baselga E, Chamlin SL, Garzon MC, Horii KA, et al. Prospective study of infantile hemangiomas: Clinical characteristics predicting complications and treatment. Pediatrics. 2006;118:3.

19. Leaute-Labreze C, Hoeger P, Mazereeuw-Hautier J, Guibaud L, Baselga E, Posiunas $\mathrm{G}$, et al. A randomized, controlled trial of oral propranolol in infantile hemangioma. N Engl J Med. 2015;372:8.

20. Sans V, de la Roque ED, Berge J, Grenier N, Boralevi F, Mazereeuw-Hautier J, et al. Propranolol for severe infantile hemangiomas: Follow-up report. Pediatrics. 2009;124:3.

21. Leaute-Labreze C. Infantile hemangiomas: The revolution of beta-blockers Rev Prat. 2014:64:10

22. Vivas-Colmenares GV, Bernabeu-Wittel J, Alonso-Arroyo V, de Cardenas JA $M$, Fernandez-Pineda I. Effectiveness of propranolol in the treatment of infantile hemangioma beyond the proliferation phase. Pediatr Dermatol. 2015;32:3.

23. Stillo F, Baraldini V, Dalmonte P, El Hachem M, Mattassi R, Vercellio G, et al. Vascular anomalies guidelines by the italian society for the study of vascular anomalies (SISAV). Int Angiol. 2015;34(2 Suppl 1):1-45.

24. Marqueling AL, Oza V, Frieden IJ, Puttgen KB. Propranolol and infantile hemangiomas four years later: A systematic review. Pediatr Dermatol. 2013;30:2.

25. Prey S, Voisard JJ, Delarue A, Lebbe G, Taieb A, Leaute-Labreze C, et al. Safety of propranolol therapy for severe infantile hemangioma. JAMA. 2016;315:4

26. Begaud B, Evreux JC, Jouglard J, Lagier G. Imputation of the unexpected or toxic effects of drugs. actualization of the method used in france. Therapie. 1985;40:2.

27. Brandling-Bennett HA, Metry DW, Baselga E, Lucky AW, Adams DM, Cordisco MR, et al. Infantile hemangiomas with unusually prolonged growth phase: A case series. Arch Dermatol. 2008;144:12.

28. Saidi W, Zaouali A, Saihi N, Mokni S, Denguezli M, Nouira R. Cardiorespiratory arrest following bronchiolitis in a child treated with propranolol for hemangioma. Ann Dermatol Venereol. 2014;141:8-9.

\section{Submit your next manuscript to BioMed Central and we will help you at every step:}

- We accept pre-submission inquiries

- Our selector tool helps you to find the most relevant journal

- We provide round the clock customer support

- Convenient online submission

- Thorough peer review

- Inclusion in PubMed and all major indexing services

- Maximum visibility for your research

Submit your manuscript at www.biomedcentral.com/submit
( BioMed Central 